Palabras clave: Trastorno mental, Tratamiento psiquiátrico, Creencias sociales.

\title{
Diferenciación en las representaciones sociales de los trastornos mentales y los tratamientos psiquiátricos
}

\author{
Danielle Goerg, sociologist* \\ Werner Fischer, sociologist* \\ Eric Zbinden, sociologist* \\ José Guimón, MD** \\ * Department of Psychiatry, University \\ Hospital of Geneva \\ ${ }^{* *}$ Honorary Professor, University of Geneva \\ SWITZERLAND \\ ** Professor of Psychiatry, University of the \\ Basque Country \\ SPAIN
}

\begin{abstract}
RESUMEN- Puesto que la representación social de la enfermedad mental y su tratamiento juegan un papel importante en su detección y en la orientación de los pacientes hacia distintas terapias, es importante determinar si la sociedad diferencia entre los distintos trastornos mentales y sus tratamientos. Estas representaciones se examinan en una encuesta realizada en una muestra estratificada de la población suiza para tres enfermedades mentales, en términos de definición como enfermedad, etiología, tratamientos previstos y pronóstico.

Existe una diferenciación clara entre esquizofrenia por un lado, y depresión y trastorno de pánico por otro. La esquizofrenia se considera con más frecuencia una enfermedad real con etiología multifactorial, un pronóstico más pesimista y para la que la psicoterapia y la terapia familiar son tratamientos recomendados. Hay, sin embargo falta de diferenciación en cuanto a los distintos factores etiológicos, en particular los factores psicosociales, que se consideran muy importantes, y ciertos tratamientos, especialmente la medicación, recomendada sólo por un cuarto de la población.
\end{abstract}

\section{Introducción}

La representación social de, y las actitudes hacia los trastornos mentales y su tratamiento juegan un papel importante, tanto en la detección como en el tratamiento del enfermo mental. Estas representaciones sociales condicionan el modo en el que los terapeutas, los 
pacientes y sus familias se ven unos a otros. Son también factores determinantes de cuán cumplimentadores serán los pacientes con el tratamiento propuesto. Ciertos estudios sobre la actitud de la población general hacia el tratamiento psiquiátrico y, en particular hacia la medicación psicotrópica, han demostrado que la sociedad apenas diferencia entre los diferentes trastornos mentales y los distintas tipos de medicación psicotrópica y sus indicaciones. Así, en Australia se observó que el mismo sistema de creencias respecto a los tratamientos adecuados se aplicaba a diversos trastornos mentales (Jorm et al. 1997a). En Alemania, la sociedad prácticamente no hace distinción entre esquizofrenia, depresión mayor y trastorno de pánico en lo concerniente al tratamiento, y se da mayor preferencia a la psicoterapia que a la medicación (Angermeyer, Daümer y Matschinger 1993, Angermeyer y Matschinger 1996a). Para Benker, Kepplinger y Sobota (1995), la mayoría de la población alemana prescinde de recurrir a la medicación psicotrópica en casos de trastorno mental. El espectro de las enfermedades mentales parece reducido a dos extremos: locura y malestar psíquico, dentro del cual la sociedad clasifica también algunos trastornos severos como la depresión. Para la locura hay una tendencia a aceptar la medicación; para el malestar psíquico la medicación se suele rechazar en general, ya que no es percibida como una enfermedad.

Otros trabajos dirigidos principalmente al reconocimiento de los trastornos psiquiátricos, sus causas y pronóstico, así como la actitud hacia los que padecen tales trastornos, han mostrado algunas veces resultados más diferenciados en la sociedad en general (Jorm et al. 1997b, 1997c, Link et al. 1999) y en muestras específicas de población (Eker 1989, Arkar \& Eker 1992). Aunque parece que se establecen pocas diferencias entre los diversos trastornos psiquiátricos, parte de estos trabajos tienden a mostrar que la esquizofrenia se percibe con más facilidad como una enfermedad y se considera a menudo que tiene consecuencias más sombrías y provoca un distanciamiento social mayor que otros trastornos.

La hipótesis examinada en nuestro estudio es que la población suiza no diferencia entre los distintos trastornos psiquiátricos ni entre los diferentes tratamientos que se pueden elegir para estos trastornos. Más específicamente, será tratada la cuestión de la falta de diferenciación entre tres trastornos psiquiátricos serios (esquizofrenia, depresión, trastornos de pánico) bajo los siguientes aspectos: su definición como enfermedad, su etiología, los tratamientos que se consideran adecuados y, finalmente, su pronóstico. Si aún así apareciesen diferencias, se investigarán los factores sociales determinantes y los elementos que pudieran estar ligados a ellas.

\section{Método}

Los resultados presentados son parte de un estudio realizado en 1997 con una muestra representativa de la población residente en Suiza, con edades comprendidas entre 20 y 75 años, tanto en la parte de habla germana como en la francófona. La muestra estratificada se basó en el sexo, la edad y la actividad profesional. Los 1.016 sujetos seleccionados contestaron a un cuestionario estandarizado que se ocupaba principalmente de la representación de los trastornos mentales y su tratamiento, la actitud hacia la medicación psicotrópica y la actitud hacia los pacientes psiquiátricos. También se examinaron las características sociodemográficas, profesionales, culturales y familiares de los sujetos.

Para esbozar las representaciones sociales de los trastornos y los tratamientos psiquiátricos, se elaboraron tres historias clínicas pre- 
sentando los siguientes tres casos: depresión mayor, esquizofrenia y trastorno de pánico. Fueron elaboradas basándose en criterios DSM-III con ayuda de un psiquiatra. Cada historia se presentó a un tercio de la muestra. Así, la historia que mostraba un caso de esquizofrenia se mostró a 353 sujetos, la de depresión mayor a 333 sujetos y la de trastornos de pánico a 330. Las tres submuestras no se diferenciaban en las principales características sociodemográficas, profesionales, culturales y familiares de los entrevistados. Tampoco fueron diferenciados según su proximidad a la psiquiatría, contactos con personas que hubieran sufrido dificultades psicológicas o su propia experiencia con tales problemas.

Tras la presentación de cada historia, preguntamos a los entrevistados cuál sería el tratamiento más útil para la persona descrita (pudiendo elegir un máximo de tres tratamientos) de una lista de seis posibilidades, y dejando otras formas de terapia abiertas a sugerencia. Para aquellos sujetos que no seleccionaban la medicación, se les planteó una pregunta semicerrada con 18 respuestas pre-codificadas referentes a los motivos por los que no la mencionaron. Estos ítems se reagruparon en seis variables de tipo presencia-ausencia. La representación social de la etiología de la enfermedad se evaluó primero a través de una lista de 18 ítems, tomados de Angermeyer y Matschinger (1994). A partir de ahí se crearon seis índices ponderados, reagrupando en cada uno tres ítems referidos a la misma dimensión: factores de estrés psicosocial (incluyendo importantes acontecimientos perturbadores de la vida, problemas laborales y dificultades familiares o de pareja), factores psicológicos (problemas psicológicos personales, falta de fuerza de voluntad y excesiva autoexigencia), factores biológicos (enfermedades cerebrales, constitución frágil, herencia), aquellos factores ligados a la socialización temprana (carencia de afecto de los padres, separación de los padres durante la infancia, padres sobreprotectores), factores sociales (percepción del distanciamiento del mundo actual respecto a la naturaleza, pérdida de la tradición, desigualdad social y explotación) factores sobrenaturales (intervención divina, astrología, brujería). Responder "sî" a cada ítem se valoraba con dos puntos, "quizá" 1 punto. Los dos ítems acerca de la definición de enfermedad y el pronóstico eran preguntas cerradas.

Se establecieron también otros índices sobre factores sociales. Estaban referidos principalmente a la diferenciación cultural (extranjeros, personas llegadas desde otras regiones, lengua materna distinta a la región de residencia y cónyuge con diferente lengua materna), un índice ponderado de proximidad a la psiquiatría (una institución psiquiátrica localizada en las cercanías, conocimiento del trabajo de una institución psiquiátrica, otros tipos de vínculo a una institución) y un índice ponderado de contactos con personas que sufren trastornos mentales.

Las respuestas a las historias fueron comparadas con las habituales pruebas de chicuadrado y análisis de varianza (además de la prueba de Scheffe), dependiendo del tipo de variable estudiada. Se utilizaron análisis de regresión logística para distinguir factores sociales asociados a la definición de los diferentes trastornos en términos de enfermedad.

\section{Resultados}

\section{Definición de enfermedad mental}

La valoración de la percepción social en las distintas historias clínicas de las personas descritas como enfermas de un trastorno psiquiátrico, era indicada en su opinión como: ciertamente enfermo, probablemente enfermo, probablemente no enfermo o ciertamente no enfermo. 
Teniendo en cuenta las respuestas positivas y aquéllas con una tendencia positiva, notamos que eran más los sujetos que consideraban mentalmente enferma la persona presentada en la historia de esquizofrenia (84\%) que aquéllos de depresión (62\%) o de trastorno de pánico (69\%), con diferencias estadísticamente significativas. Esta distinción era más marcada para la respuesta inequívocamente positiva (38,5\% para esquizofrenia, 21,3\% para depresión, $\mathrm{p}<0,001$; $18,8 \%$ para trastorno de pánico, $\mathrm{p}<0,001)$.

\section{Etiología de los trastornos psiquiátricos}

Preguntados acerca de los factores que podían estar en el origen de la situación presentada en la historia clínica, la sociedad, por encima de todo, favorecía los factores de estrés psicosocial y los factores psicológicos (Tabla I). Los factores biológicos, los ligados a la socialización temprana y los factores sociales se mencionaban con menor frecuencia como causas de la enfermedad psiquiátrica y los factores sobrenaturales se citaban sólo excepcionalmente.

Los sujetos no diferenciaron entre el estrés psicosocial y los factores sociales en las historias. Los casos clínicos inspiraron, sin embargo, representaciones contrastadas de su etiología para los otros tres tipos de fac- tores. Así, la representación social de la etiología de la esquizofrenia se distinguía por el mayor peso otorgado a los factores ligados a la socialización que para los otros dos diagnósticos. Los factores biológicos se nombraban con más frecuencia que para la depresión y los factores psicológicos más que para el trastorno de pánico.

Aparecieron muy pocas diferencias en la representación de la etiología de la depresión y del trastorno de pánico, excepto en el peso de los factores psicológicos, mayor en el primer caso, y el de los factores biológicos en el segundo.

\section{Tratamientos considerados}

Los sujetos tenían que elegir tres tratamientos considerados más beneficiosos para la persona descrita en la historia. Además de las soluciones de tipo psiquiátrico, la lista mencionaba el posible recurso a medios alternativos o naturales.

Los enfoques psiquiátricos eran, con mucho, los preferidos y la psicoterapia individual aparecía con el tratamiento más apropiado para los trastornos mentales (Tabla II). La terapia de relajación se consideraba útil, pero en menor grado, y lo mismo ocurría con la medicación psicotrópica, la terapia familiar, y los productos o métodos naturales, además de la meditación el yoga.

Tabla I

Factores etiológicos de los trastornos psiquiátricos (media de los índices comprimidos entre 0 y 6)

\begin{tabular}{|c|c|c|c|c|c|c|c|c|}
\hline Factores & Total & Esquizofrenia & Depresión & $\begin{array}{c}\text { Trastorno de } \\
\text { pánico }\end{array}$ & $\begin{array}{c}\text { Análisis de } \\
\text { varianza }\end{array}$ & $\mathrm{E}^{1} \mathrm{D}^{1}$ & Scheffe (dif. signif.) & $\begin{array}{l}\text { D/TP } \\
\text { if.) }\end{array}$ \\
\hline Estrés psicosocial & 3,4 & 3,3 & 3,3 & 3,5 & & & & \\
\hline Psicológicos & 3,1 & 3,2 & 3,1 & 2,8 & $* * * 2$ & & $*$ & $*$ \\
\hline Biológicos & 2,2 & 2,3 & 2,0 & 2,3 & $* *$ & $*$ & & $*$ \\
\hline $\begin{array}{l}\text { Ligados a la } \\
\text { socialización }\end{array}$ & 2,1 & 2,4 & 2,0 & 1,7 & $* * *$ & $*$ & $*$ & \\
\hline Sociales & 2,1 & 2,2 & 2,0 & 2,0 & & & & \\
\hline
\end{tabular}

( ${ }^{1}$ Las respuestas comparan una enfermedad psiquiátrica con otra (en este caso esquizofrenia vs. depresión).

$\left({ }^{2}\right)$ La probabilidad se indica como sigue: * $\mathrm{p}<0,05 ; * * \mathrm{p}<0,01 ; * * * \mathrm{p}<0,001$. 
Otras posibilidades fueron raramente sugeridas y los sujetos que decían que la persona descrita no necesitaba ningún tipo de tratamiento fueron excepcionales.

Con la excepción de la medicación, que fue mencionada por un cuarto de los sujetos para los tres tipos de trastorno psiquiátrico, se encontraron variaciones muy significativas para los tratamientos elegidos para la esquizofrenia por un lado, y la depresión y el trastorno de pánico por el otro. Para la esquizofrenia, la psicoterapia se consideraba particularmente importante, seguida de la terapia familiar y luego la medicación. El rango de tratamiento se presentaba más diversificado para la depresión y el trastornos de pánico, con la relajación y los tratamientos alternativos citados con más frecuencia que para la esquizofrenia.

Hubo varias diferencias entre los tratamientos seleccionados para la depresión y el trastorno de pánico.

\section{Razones para no elegir la medicación}

Las principales razones alegadas para no elegir la medicación fueron las mismas para los tres casos clínicos considerados: dudas sobre la efectividad de la medicación $(45,6 \%$ de los que no la eligieron) y miedo a los efec- tos secundarios $(43,4 \%)$. Otras razones, que tenían que ver con las características de las personas presentadas en las historias, que supuestamente no necesitaban medicación $(30,5 \%)$ o no estaban enfermas (24\%), o con actitudes negativas hacia la medicación en general, fueron menos citadas. Muy pocos sujetos afirmaron no estar familiarizados con la medicación psiquiátrica $(4,6 \%)$.

\section{Pronóstico de los trastornos psiquiátricos}

Preguntados sobre la evolución que la persona de la historia tendría de seguir el tratamiento sugerido, los sujetos dieron un pronóstico relativamente favorable. Más de 9 sujetos de cada $10(94,4 \%)$ pensaban que la persona podría curarse o mejorar, aunque sólo fuera parcialmente, fuera cual fuese el diagnóstico en cuestión. Sin embargo, si nos limitamos a la "posibilidad de curación", sólo el $24,7 \%$ de los sujetos entrevistados la consideraba probable para la esquizofrenia, el $34,4 \%$ para la depresión $(\mathrm{p}<0,05)$ y el $44,9 \%$ para el trastorno de pánico ( $\mathrm{p}<0,001)$. El pronóstico para la depresión era menos favorable que el del trastorno de pánico $(\mathrm{p}<$ $0,05)$. Los sujetos que definían el trastorno como una enfermedad tendían a pensar con menor frecuencia que podía ser curado.

Tabla II

Tratamientos propuestos para trastornos psiquiátricos: Porcentaje de nominaciones favorables

\begin{tabular}{|c|c|c|c|c|c|c|c|}
\hline Tratamientos & $\begin{array}{c}\text { Total } \\
\%\end{array}$ & $\begin{array}{c}\text { Esquizofrenia } \\
\%\end{array}$ & $\begin{array}{c}\text { Depresión } \\
\%\end{array}$ & $\begin{array}{c}\text { Trastorno de } \\
\text { pánico } \%\end{array}$ & $\mathrm{E} / \mathrm{D}^{1}$ & $\begin{array}{c}\mathrm{E} / \mathrm{TP} \\
\chi^{2}\end{array}$ & $\mathrm{D} / \mathrm{TP}$ \\
\hline Psicoterapia & 56,6 & 67,7 & 50,5 & 50,9 & $* * * 2$ & $* * *$ & \\
\hline Terapia de relajación & 36,4 & 15,6 & 45,6 & 49,4 & $* * *$ & $* * *$ & \\
\hline Medicación & 25,7 & 26,9 & 22,8 & 27,3 & & & \\
\hline Terapia familiar & 22,1 & 38,5 & 16,5 & 10,3 & $* * *$ & $* * *$ & $*$ \\
\hline Medios naturales & 20,8 & 11,0 & 29,7 & 22,1 & $* * *$ & $* * *$ & $*$ \\
\hline Meditación, yoga & 20,3 & 14,4 & 27,6 & 19,1 & $* * *$ & & $*$ \\
\hline Otros tratamientos & 12,5 & 7,6 & 10,5 & 19,7 & & $* * *$ & $* *$ \\
\hline
\end{tabular}

( ${ }^{1}$ Las respuestas comparan una enfermedad psiquiátrica con otra (en este caso esquizofrenia vs. depresión).

$\left({ }^{2}\right)$ La probabilidad se indica como sigue: $* \mathrm{p}<0,05 ; * * \mathrm{p}<0,01$; *** $\mathrm{p}<0,001$. 


\section{¿Qué podría explicar la falta de reconocimiento de los trastornos mentales?}

La mayoría de los entrevistados consideraban que los casos presentados en las historias mostraban algún tipo de trastorno mental, especialmente para la esquizofrenia. Consideramos importante investigar qué factores están ligados a la ausencia de reconocimiento del trastorno como enfermedad psiquiátrica y a qué otros aspectos de la representación social afecta esta falta de reconocimiento.

Hicimos una regresión logística, para cada una de las historias clínicas, para relacionar la falta de reconocimiento de la enfermedad con las características sociodemográficas (sexo, edad), socioculturales (nivel profesional, distanciamiento cultu- ral), proximidad a la psiquiatría y contactos con personas que sufren trastornos psiquiátricos. La probabilidad de no definir la esquizofrenia como una enfermedad fue diferente para hombres y mujeres. Para los hombres no había relación con ninguna otra variable, pero para las mujeres la falta de reconocimiento estaba ligada a una edad más joven y a un nivel sociolaboral más bajo (Tabla III).

Es más, la ausencia de definición de la esquizofrenia como enfermedad se correspondía con distintas representaciones de su etiología y tratamiento. Las mujeres que no identificaban un trastorno psiquiátrico en la historia sugerían con menor frecuencia una etiología de naturaleza biológica o psicológica. En lo que al tratamiento se refiere, el uso de psicoterapia $(51,9 \%$ vs. $77,6 \%$, p < 0,01) y

Tabla III

Regresión logística sobre la ausencia de definición de esquizofrenia como una enfermedad (mujeres)

\begin{tabular}{lcc} 
Variables & O. R. & $\mathrm{p}$ \\
\hline Edad & $-0,0600$ & 0,0074 \\
Nivel profesional & $-0,6317$ & 0,0168 \\
Diferenciación culturales & $-0,0734$ & 0,7368 \\
Proximidad a la psiquiatría & $-0,0962$ & 0,2985 \\
Contactos con personas que sufren un trastorno psiquiátrico & $-0,0792$ & 0,5727 \\
\hline
\end{tabular}

medicación $(11,1 \%$ vs. $36,0 \%$, p < 0,05\%) se consideraban mucho menos pertinentes. Ninguno de los factores considerados en la regresión logística parecían intervenir en la valoración de la depresión y el trastorno de pánico. Encontramos, sin embargo, diferencias con respecto a las representaciones en su etiología y tratamiento. Cuando la depresión no era percibida como una enfermedad, los factores sociales se mencionaban con menor frecuencia en su etiología y la psicoterapia, además de la medicación, eran menos sugeridas como tratamiento. Para el trastorno de pánico, los factores ligados a la temprana socialización se citaban menos y, por el contrario, si la psicoterapia se mencionaba menos, otros tratamientos (principalmente de naturaleza somática) eran elegidos con mayor frecuencia.

\section{Discusión}

Hay varias similitudes y varias diferencias en las representaciones sociales que la gente tiene de la definición, etiología, trata- 
miento y consecuencias de los trastornos psiquiátricos.

1. Investigando los aspectos de la diferenciación, documentamos que la principal diferencia se encuentra en las representaciones opuestas que la población tiene de la esquizofrenia, comparada con la depresión y el trastorno de pánico.

En Suiza, la esquizofrenia se percibe más a menudo como una enfermedad que la depresión y el trastorno de pánico, por lo que se confirman los resultados de Link et al. (1999), que compararon cinco historias en EE.UU. y Jorm et al. (1979b), que examinaron el reconocimiento de la esquizofrenia y la depresión entre la población australiana. Sus pronósticos se mostraban también más pesimistas (Jorm et al. 1979b, Arkar \&Eker 1992). Si nos fijamos en la etiología, hay un respaldo todavía más pronunciado de la explicación multifactorial en favor de la esquizofrenia, comparado con la depresión y el trastorno de pánico. Ésta es más frecuentemente percibida que los otros dos diagnósticos como ligada a la socialización temprana y los factores biológicos pesan más que en la depresión. Los australianos también conceden más importancia a los sucesos infantiles y a factores genéticos en el caso de esquizofrenia que en el de depresión (Jorm et al. 1997c) y en Alemania, los factores biológicos son considerados más importantes para la esquizofrenia que para la depresión y el trastorno de pánico (Angermeyer \& Matschinger 1999). Finalmente, en nuestro estudio, los factores psicológicos se mencionan más a menudo para la esquizofrenia que para el trastorno de pánico. Entre los factores psicológicos mencionados, la falta de fuerza de voluntad y la escasa conducta de auto-exigencia parecen indicar la existencia, en este caso, de un tipo de juicio moral. Atendiendo al tratamiento, la gente masivamente favorece dos tipos de tratamiento psiquiátrico: la psicoterapia individual y la terapia familiar. Esto podría estar asociado con el tipo de representación etiológica efectuada para este trastorno. Jorm et al. (1997a) también observaron que las intervenciones sobre el estilo de vida (naturopatía, actividad física, amigos, etc.) recibían clasificaciones menos positivas para la historia de la esquizofrenia que para la depresión. Existen importantes diferencias en las representaciones de la gente entre esquizofrenia y depresión o trastorno de pánico para todos los tratamientos propuestos, con excepción de la medicación, que comentaremos más adelante.

En contraste, las representaciones sociales de la depresión y el trastorno de pánico son muy poco diferenciadas. Estos trastornos son menos a menudo considerados enfermedades que la esquizofrenia, aunque para una importante parte de los entrevistados no se excluye dicha posibilidad. Para una proporción no diferenciada de sujetos, la depresión no parece vista como una enfermedad, sino como una situación bastante común. La representación del trastorno de pánico es indudablemente más vaga y a veces podría asociarse a problemas somáticos, por lo que la gente es más favorable a la causalidad psicológica de la depresión. Los tratamientos propuestos son a menudo los mismos, dando incluso la gente preferencia a los tratamientos alternativos para la depresión y a otros métodos, frecuentemente de naturaleza somática, para el trastorno de pánico. Finalmente, el pronóstico para este trastorno es discretamente más favorable que para la depresión.

El abismo entre la esquizofrenia por un lado, y la depresión y el trastorno de pánico o por otro, aparece evidente. Esta divergencia indudablemente surge de los elementos extraños de conducta y la no conformidad social con el trastorno esquizofrénico, obviamente más presente que en el otro cuadro clínico. Diferentes estudios que tratan de la distancia 
social hacia la enfermedad mental tienden a confirmar esta hipótesis. Se documenta que la distancia social deseable varía de acuerdo con el tipo de trastorno psiquiátrico. La mayor distancia social se observa hacia los alcohólicos y los drogadictos, luego hacia los pacientes esquizofrénicos, mientras que los individuos que sufren de depresión mayor encuentran la mayor aceptación social. La distancia social está directamente relacionada con la percepción de peligrosidad (Link et al. 1999).

2. Es cierto que más allá de las diferencias examinadas en este punto, hay otros aspectos de la representación social que tienden más hacia la falta de diferenciación. Esto principalmente concierne a la etiología de los trastornos psiquiátricos y tratamientos.

En la representación social de la etiología de los trastornos, se le da un peso considerable a los factores psicosociales, cualquiera que sea el diagnóstico que se considere. Los factores sociales, aunque de manera más débil, casi no varían. La gran importancia dada a los factores psicosociales aparece en diferentes países. En EE.UU., las circunstancias estresantes se consideran como la primera causa de varios trastornos psiquiátricos (Link et al. 1999) y en Australia, el factor citado más a menudo como causa tanto de la esquizofrenia como de la depresión se hallan en el entorno social más inmediato (Jorm et al. 1997c). Angermeyer y Matschinger (1999) también subrayaron el énfasis dado por la sociedad a los factores psicosociales, en particular para la esquizofrenia, un énfasis hasta el punto de que las ideas acerca del estrés se han ido propagando entre la sociedad en general.

Desde el punto de vista de los tratamientos recomendados para los diferentes trastornos psiquiátricos, debemos primero señalar la importancia dada a la psicoterapia. A pesar de las diferencias de acuerdo con la evidencia diagnóstica descrita previamente, aparece como el tratamiento preferido para los trastornos mentales, lo que se corresponde con los resultados de diferentes estudios realizados en Alemania. La predominancia de la psicoterapia entre los tratamientos parece confirmar la amplia difusión de las nociones psicológicas entre la gente.

En contraste, el público da una menor importancia a la medicación, independientemente del trastorno, incluso si lo clasifica en tercer lugar entre los tratamientos propuestos. Sólamente una cuarta parte de los sujetos propone recurrir a la medicación, tanto para la esquizofrenia como para la depresión y para el trastorno de pánico. Jorm et al. (1997a) muestran que los australianos tienen una visión negativa de los tratamientos médi$\cos$ (que incluyen diferentes tipos de medicación, pero también terapia electroconvulsiva y hospitalización psiquiátrica), mientras que es favorable a las intervenciones psicológicas o ligadas al estilo de vida. Benker et al. (1995) obtuvieron más resultados favorables con la farmacoterapia para la esquizofrenia que para los otros seis trastornos examinados, mientras que Angermayer, Daümer y Matschinger (1993), como en nuestro estudio, informaron de la misma ausencia de discriminación. No obstante, la posición atribuída a la farmacoterapia es todavía más débil que en nuestros resultados, donde sólo el 14\% de la población es favorable. No podemos, sin embargo, saber si esta diferencia está ligada al método utilizado, a diferencias entre la población alemana y suiza $\mathrm{o}$, finalmente, a una evolución de los juicios en el tiempo, ya que esas encuestas se llevaron a cabo en 1990 y las nuestras en 1997. Estudiando las actitudes hacia la medicación psicotrópica, Fisher et al. (1999) documentaron una muy clara relación entre las actitudes hacia los fármacos psicotrópicos y el nivel sociocultural, con los niveles altos sociales y culturales asociados a las actitudes más favorables. 
Entre las razones dadas para no elegir la medicación, el público especialmente expresa dudas sobre su efectividad y miedo a los efectos secundarios. Estas razones no varían en función del caso clínico previsto, con excepción de una ligera mejor tolerancia a sus efectos secundarios para la esquizofrenia. Para Angermeyer, Daümer y Matschinger (1993), el público no diferencia entre las diferentes categorías de psicotrópicos y extrapolan las propiedades de los tranquilizantes a los psicotrópicos en general.

\section{Conclusión}

En resumen, surgen puntos de vista contrastados del público acerca de los trastornos mentales, mostrando una clara diferenciación entre esquizofrenia, por un lado y depresión y trastorno de pánico por el otro; una falta de discriminación para algunos factores etiológicos y el empleo de medicación. Algunos resultados sugieren una cierta ambigüedad en las representaciones, particularmente en la percepción de los trastornos como enfermedades y su pronóstico. Serían necesarias investigaciones posteriores desde una aproximación más cualitativa para precisar esas representaciones. Los resultados también demuestran lo que Jorm (2000) llamó una "pobre alfabetización en salud mental". El público muestra una cierta falta de conciencia del actual estado de conocimiento, o más específicamente, de la opinión de los expertos actualmente dominante en este área. Parece, sin embargo, que algunos argumentos procedentes del actual debate científico (o a veces de algunos debates cercanos) han sido incorporados dentro del "punto de vista del sentido común". Podemos explicar así la importancia acordada para los factores psicosociales en la etiología de los trastornos mentales o el peso de la psicoterapia individual en el tratamiento de los trastornos mentales. La psicología popular ha impregnado a una amplia proporción de la gente y se utilizan ciertos elementos para describir trastornos que en ocasiones no parecen ser serios. Esto es particularmente cierto para la depresión y, en cierto grado, para el trastorno de pánico.

Frente a la historia clínica que presenta un caso de esquizofrenia, otras características pueden jugar un papel relevante: aquéllas que se refieren a una conducta extraña, indicadora de la locura. Sin embargo, para un segmento de la población este trastorno no se percibe como una enfermedad. Para ellos, la cualidad de ser diferente de la desviación no está lastrada por connotaciones psiquiátricas. Para los hombres, esta ausencia de reconocimiento no está ligada a características sociales ni a sus experiencias psiquiátricas. Es como si el trastorno sólo pudiera ser entendido en función de alguna lógica desconocida que nuestros datos no revelan. Por otro lado, las mujeres de mayor edad y aquéllas con más altas cualificaciones profesionales parecen ser las que mejor identifican la enfermedad. Por analogía con lo mostrado por Boltanski (1971) (la percepción de las sensaciones de morbilidad varía entre las clases sociales y las mujeres prestan más atención a sus cuerpos que los hombres), podemos suponer que la misma lógica puede aplicarse a la percepción de la salud mental en los otros. La mujeres, que generalmente son las responsables del cuidado de la salud de otros (hijos, familia) podrían adquirir a través de las experiencias vitales una mayor capacidad para percibir signos patológicos.

Debemos subrayar un último punto. Estas representaciones son las de una población que, en su mayoría, puede haber tenido cierta experiencia en el campo de la Psiquiatría o la Psicología. Cerca de dos tercios indican que algunos miembros de su familia, amigos o conocidos han sufrido dificultades psicológi- 
cas y que algunos de ellos han sido tratados por un psicólogo o psiquiatra; en casi la mitad, alguna persona de su entorno ha sido ingresada en un hospital psiquiátrico; finalmente, una cuarta parte de los sujetos entrevistados dicen que ellos mismos han sufrido dificultades psicológicas, aunque los que buscaron ayuda por este motivo son menos. Parecería, por lo tanto, que aunque la experiencia personal con la enfermedad mental podría modificar las actitudes hacia los pacientes psiquiátricos (Angermeyer y Matschinger 1996b, y en otros resultados de nuestro estudio), tales experiencias tienen poco efecto en las representaciónes y conocimiento de la gente.

\section{Bibliografía}

ANGERMEYER, M.C., DAUMER, R., MATSCHINGER, H. Benefits and risks of psychotropic medication in the eyes of the general public: Results of a survey in the Federal Republic of Germany. Pharmacopsychiatry 26, 114120, 1993.

ANGERMEYER, M.C., MATSCHINGER, H. Lay beliefs about schizophrenic disorder: the results of a population survey in Germany. Acta Psychiatrica Scandinavica 89 (suppl. 382), 39-45, 1994.

ANGERMEYER, M.C., MATSCHINGER, H. Public attitudes towards psychiatric treatment. Acta Psychiatrica Scandinavica 94, 326-336, 1996 a.

ANGERMEYER, M.C., MATSCHINGER, H. The effect of personal experience with mental illness on the attitude towards individuals suffering from mental disorders. Social Psychiatry and Psychiatric Epidemiology 31, 321-326, 1996b.

ANGERMEYER, M.C., MATSCHINGER, H. Social representations of mental illness among the public, 20-28. In. Guimón J., Fischer W., Sartorius N. (eds): The image of madness. The public facing mental illness and psychiatric treatment. Basel: Karger, 1999.

ARKAR, H., EKER, D. Influence of having a hospitalized mentally ill member in the family on attitudes toward mental patients in Turkey. Social Psychiatry and Psychiatric Epidemiology 27, 151-155, 1992.
BENKERT, O., KEPPLINGER, H.M., SOBOTA, K. Psychopharmaka im widerstreit. Eine studie zur akzeptanz von psychopharmaka - bevolkerungsumfrage und medienanalyse, Berlin: Springer, 215 p, 1995.

BOLTANSKI, L. Les usages sociaux du corps. Annales 26(1), 205-233,1971.

EKER, D. Altitudes toward mental illness: recognition, desired social distance, expected burden and negative Influence on mental health among Turkish freshmen. Social Psychiatry and Psychiatric Epidemiology 24, 146-150, 1989.

FISCHER, W., GOERG, D., ZBINDEN, E., GUIMÓN, J. Determining factors and the effects of attitudes towards psychotropic medication, 162-186. In Guimón J., Fischer W., Sartorius N. (eds): The image of madness. The public facing mental illness and psychiatric treatment. Basel: Karger, 1999.

JORM, A.F. Mental health literacy. Public knowledge and beliefs about mental disorders. The British Journal of Psychiatry 177, 396-401, 2000.

JORM, A.F., KORTEN, A.E., RODGERS, B., POLLITT, P., JACOMB, P.A., CHRISTENSEN, H., JIAO, Z. Belief systems of the general public concerning the appropriate treatments for mental disorders. Social Psychiatry and Psychiatric Epidemiology 32, 468-473, 1997a.

JORM, A.F., KORTEN, A.E., JACOMB, P.A., CHRISTENSEN, H., RODGERS, B., POLLITT, P. Mental health literacy: a survey of the public's ability to recognise mental disorders and their beliefs about the effectiveness of treatment. The Medical Journal of Australia 166, 182-186, $1997 b$.

JORM, A.F., KORTEN, A.E., JACOMB, P.A., CHRISTENSEN, H., RODGERS, B., POLLITT, P. Public beliefs about causes and risk factors for depression and schizophrenia. Social Psychiatry and Psychiatric Epidemiology 32, 143-148, 1997c.

LINK, B.G., PHELAN, J.C., BRESNAHAN, M., STUEVE, A., PESCOLIDO, B.A. Public conceptions of mental illness: Labels, causes, dangerousness, and social distance. American Journal of Public Health, 89(9), 13281333, 1999.

Dirección para correspondencia:

D. Goerg, Département de psychiatrie

2, Chemin du Petit-Bel-Air

CH- 1225 Chêne-Bourg

Geneva, Switzerland

E-mail: Danielle.Goerg@hcuge.ch 